\section{Case Reports in Ophthalmology}

\title{
Five-Port Combined Limbal and Pars Plana Vitrectomy for Infectious Endophthalmitis
}

\author{
Kunyong $\mathrm{Xu}^{\mathrm{a}}$ Eric K. Chin ${ }^{\mathrm{b}}$ David R.P. Almeida ${ }^{\mathrm{c}}$ \\ ${ }^{a}$ Department of Ophthalmology, Queen's University, Kingston, ON, Canada; ${ }^{b}$ Retina \\ Consultants of Southern California, Redlands, CA, USA; 'VitreoRetinal Surgery, PA, \\ Minneapolis, MN, USA
}

\section{Keywords}

Limbal vitrectomy $\cdot$ Pars plana vitrectomy $\cdot$ Infectious endophthalmitis

\begin{abstract}
Pars plana vitrectomy for acute infectious endophthalmitis can be challenging due to severe inflammation in the anterior chamber creating significant media opacity. We describe a surgical technique combining limbal based vitrectomy and pars plana vitrectomy to manage acute infectious endophthalmitis. Limbal based vitrectomy facilitates removal of anterior chamber fibrin and inflammatory membranes for safe and optimal posterior pars plana vitrectomy.

(C) 2016 The Author(s) Published by S. Karger AG, Basel
\end{abstract}

\section{Introduction}

Infectious endophthalmitis is a devastating vision-threatening condition consisting of inflammation of the entire globe and its intraocular contents. Surgical intervention, such as pars plana vitrectomy (PPV), has been used for cases with infectious endophthalmitis whose visual acuity is worse than light perception after cataract surgery [1]. PPV for infectious endophthalmitis is challenging given the severe inflammation in the anterior chamber which creates significant media opacity and limits visualization of the posterior pole. 


\section{Case Reports in Ophthalmology}

\begin{tabular}{l|l}
\hline DOI: $10.1159 / 000453524$ & (c) 2016 The Author(s). Published by S. Karger AG, Basel \\
\hline
\end{tabular} www.karger.com/cop

Xu et al.: Five-Port Combined Limbal and Pars Plana Vitrectomy for Infectious Endophthalmitis

Limbal based anterior vitrectomy is sometimes used by our anterior segment colleagues in cases of posterior capsule rupture and vitreous prolapse. Additionally, pediatric vitreoretinal cases involving vitreous hemorrhages, retina detachment, and opacified vitreous with uveitis benefit from Iimbal vitrectomy [2]. This surgical technique shows good efficacy and safety that is designed to avoid disruption of the vitreous base and the anterior retina [2].

Here, we describe a surgical technique combining limbal based vitrectomy and PPV to manage infectious endophthalmitis. The limbal based vitrectomy is used to initially remove infectious and inflammatory material from the anterior chamber. It is an ideal technique for visualization of the posterior pole for more complete PPV as an early intervention for infectious endophthalmitis.

\section{Surgical Technique}

Our surgical technique includes a five-trocar setup using 3 standard 25-gauge pars plana trocar/cannulas and 2 limbal anterior trocar/cannulas. Undiluted aqueous and vitreous biopsy can be obtained prior to turning the infusion on, given the multiple trocar availability.

The anterior infusion is initially turned on once it is verified in the anterior chamber via the corneal limbus. The second anterior corneal limbus cannula is used for anterior chamber washout and removal of the fibrin and inflammatory membranes. Both the vitreous cutter and retinal forceps can be used to remove fibrin membranes from the pupil margins in aphakic cases and over the intraocular lens in pseudophakic cases.

After all anterior chamber material is cleared and media clarity optimized, the posterior segment is better visualized. The infusion line of a balanced saline solution is moved to the pars plana cannula and a complete posterior vitrectomy is performed. In cases where the media opacity remains significant, the posterior vitrectomy can be performed with the anterior infusion line. At the conclusion of the case, the small gauge limbus wounds can be hydrated or closed with single interrupted 10-0-nylon sutures. Any leaking sclerotomies should be closed in a similar manner with 7-0-vicryl suture (online suppl. Video 1; for all online suppl. material, see www.karger.com/doi/10.1159/000453524).

\section{Discussion}

Patients with infectious endophthalmitis who undergo early vitrectomy might have superior outcomes with fewer complications [3-6]. However, early vitrectomy for infectious endophthalmitis might be challenging due to difficult visualization secondary to media opacity secondary to severe inflammation in the anterior chamber.

Our surgical technique of five-port combined limbal and PPV for infectious endophthalmitis allows for removal of infectious and inflammatory material from both the anterior chamber and vitreous cavity in a safe and efficient manner. The limbal based approach greatly aids visualization and potentially improves the efficacy of the PPV in cases where media opacity precludes securing a posterior segment infusion. This is significantly beneficial at removing infectious material and vitreous debris, which is paramount to accelerating infection clearance and optimizing visual outcomes.

The 25-gauge trocar/cannula limbal system provides a small-gauge easy access port in the anterior chamber with good efficacy and safety. Similar to the 23-gauge limbal approach, no intraoperative or postoperative complications were observed, such as corneal wound 
dehiscence, Descemet membrane tears, iris incarceration, epithelial downgrowth, or corneal dellen, retinal tear or dialysis, retinal detachment, suprachoroidal hemorrhage or effusion [2]. The smaller gauge cannulas (with or without valves) often allow for the entire procedure to be sutureless. If the cornea/limbal wounds are not water tight at the end of the case, they can be hydrated with a balanced saline solution on a blunt cannula.

In cases where a pars plana infusion still cannot be visualized and verified to be in the vitreous cavity, posterior vitrectomy can be performed with the limbal (anterior) infusion cannula [2]. The anterior infusion line provides adequate intraocular pressure control regardless of lens status; however, it may cause corneal striae that could degrade the view of the posterior pole. This can be minimized by ensuring that the infusion line is taped in a radial manner (i.e. perpendicular to the circumferential cornea). When the view is sufficient posteriorly, we elect to move the infusion source to the pars plana cannula as soon as able. Collectively, this surgical technique allows for safe, efficient, and complete anterior and posterior vitrectomy for infectious endophthalmitis.

\section{Statement of Ethics}

This case report was in full adherence to the Declaration of Helsinki and all Federal and State laws.

\section{Disclosure Statement}

The authors have no conflicts of interest to disclose.

\section{References}

1 Endophthalmitis Vitrectomy Study Group: Results of the Endophthalmitis Vitrectomy Study. A randomized trial of immediate vitrectomy and of intravenous antibiotics for the treatment of postoperative bacterial endophthalmitis. Arch Ophthalmol 1995;113:1479-1496.

2 Kay CN, Quiram PH, Mahajan VB: 23-gauge pediatric vitrectomy using limbus-based trocar-cannulas. Retina 2012;32:1023-1027.

-3 Martínez-Vázquez C, Fernández-Ulloa J, Bordón J, Sopeña B, de la Fuente J, Ocampo A, Rubianes M: Candida albicans endophthalmitis in brown heroin addicts: response to early vitrectomy preceded and followed by antifungal therapy. Clin Infect Dis 1998;27:1130-1133.

4 Birnbaum FA, Gupta G: The role of early vitrectomy in the treatment of fungal endogenous endophthalmitis. Retin Cases Brief Rep 2016;10:232-235.

-5 Sallam A, Taylor SR, Khan A, McCluskey P, Lynn WA, Manku K, Pacheco PA, Lightman S: Factors determining visual outcome in endogenous Candida endophthalmitis. Retina 2012;32:1129-1134.

-6 Yoon YH, Lee SU, Sohn JH, Lee SE: Result of early vitrectomy for endogenous Klebsiella pneumoniae endophthalmitis. Retina 2003;23:366-370. 